\title{
INFORMATION FOR AUTHORS
}

The Bulletin of the Australian Mathematical Society aims at quick publication of original research in all branches of mathematics. To ensure speedy publication, only articles which are sufficiently well presented, able to be published without revision, and which are judged by the Editor (often in consultation with an Associate Editor) to be competitive are refereed. This policy is in the interests of authors, as a quick rejection is better than a slow rejection. The Bulletin receives more than five times the material that can be published, therefore there are many commendable papers not accepted. Editorial decisions on acceptance or otherwise are taken quickly, normally within a month of receipt of the paper. Papers are accepted only after peer review.

Manuscripts are accepted for review with the understanding that the same work is not concurrently submitted elsewhere. For a paper to be acceptable for publication, not only should it contain new and interesting results, but also

(i) the exposition should be clear and attractive, and

(ii) the manuscript should be in publishable form, without revision.

Further information regarding these requirements may be found through our website www.austms.org.au/Bulletin. Authors are asked to avoid, as far as possible, the use of mathematical symbols in the title.

Articles should be prepared in LATEX using $\mathcal{A}_{\mathcal{M}} \mathcal{S}$-LATEX packages and submitted as a PDF file via our journal management system, at www.austms.org.au/Publications/Submissions/BAustMS. This permits authors to track their papers through the editorial process. Recent versions of $\mathrm{T}_{\mathrm{E}} \mathrm{X}$ are able to produce PDF files directly. A $\mathrm{LT}_{\mathrm{E} X} \mathrm{X}$ class file for the Bulletin can be downloaded from the website. Authors who need assistance may email the secretary of the Bulletin at jams@ms.unimelb.edu.au.

Authors are advised to keep copies of all files of the submitted article; the Bulletin will not accept responsibility for any loss.

\section{EDITORIAL POLICY}

1. References. Arrange references alphabetically (by surname of the first author) and cite them numerically in the text. Ensure the accuracy of the references: authors' names should appear as in the work quoted. Include in the list of references only those works cited, and avoid citing works which are in preparation or submitted. Where the work cited is not readily accessible (for example, a preprint) a copy of the article should be included with your submission.

\section{Abstracts.}

1. Each paper must include an abstract of not more than 150 words, which should contain a brief but informative summary of the contents of the paper, but no inessential details.

2. The abstract should be self-contained, but may refer to the title.

3. Specific references (by number) to a section, proposition, equation or bibliographical item should be avoided.

3. Subject Classification and Key Words. Authors should include a few key words and phrases and one or more classification numbers, following the American Mathematical Society 2010 Mathematics Subject Classification for all codes. Details of this scheme can be found on the web at www.ams.org/msc.

4. Abstracts of PhD Theses. The Bulletin endeavours to publish abstracts of all accepted Australasian $\mathrm{PhD}$ theses in mathematics. One restriction, however, is that the abstract must be received by the Editor within six months of the degree being approved.

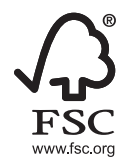

This journal issue has been printed on FSC-certified paper and cover board. FSC is an independent, non-governmental, not-for-profit organisation established to promote the responsible management of the world's forests. Please see www.fsc.org for information. 
Sums of distinct integral squares in $\mathbb{Q}(\sqrt{2}), \mathbb{Q}(\sqrt{3})$ and $\mathbb{Q}(\sqrt{6})$

Kim, J. Y. Eं Lee, Y. M.

The classification of some modular Frobenius groups

Fan, J., Du, N. E Zeng, J.

The invariants field of some finite projective linear group actions

Chen, $Y$.

Small essential spectral radius perturbations of operators with topological uniform descent

Zeng, Q., Zhong, H. E् Wu, Z.

On tensor products of weak mixing vector sequences and their applications to uniquely $E$-weak mixing $C^{*}$-dynamical systems

Mukhamedov, $F$.

Binding number and minimum degree for fractional $(k, m)$-deleted graphs

Zhou, S., Bian, Q. $\mathcal{E}^{2} \mathrm{X}$, L.

Two approaches to Möbius inversion

Huang, I-C.

Sumsets and difference sets containing a common term of a sequence

Yang, Q.-H. Ẽ Chen, Y.-G.

The boundary volume of a lattice polytope

Hegedüs, G. Ë Kasprzyk, A. M.

Rational points on three superelliptic curves

Shen, Z. E Cai, T.

Note about Lindelöf $\boldsymbol{\Sigma}$-spaces $\boldsymbol{v} \boldsymbol{X}$

Kakol, J. Ev López-Pellicer, $M$.

A new construction for pooling designs

Jin, F., Zhou, H. Eं Xu, J.

Jensen type inequalities for $\boldsymbol{Q}$-class functions

Moslehian, M. S. E Kian, $M$.

Essential commutativity of some integral and composition operators

Zhou, Z.-H., Zhang, L. Ev Zeng, H.-G.

Power-free values of the polynomial $t_{1} \cdots t_{r}-1$

Le Boudec, P.

On the degree distance of some composite graphs

Hua, $H$.

\section{Corrigendum/Erratum}

Correction to 'Decomposing linear transformations'

Wang, L. EV Zhou, $Y$.

Abstracts of Australasian PhD theses

Domain perturbation for parabolic equations

Sa Ngiamsunthorn, $P$. 